\title{
Comparison of KAtex, Bone Marrow Aspiration and DAT for the Diagnosis of Visceral Leishmaniasis
}

\author{
Ishrat Sharmin ${ }^{1}$, AKM Quamruzzaman ${ }^{2}$, Rezina parveen ${ }^{3}$, Md. Abdullah Yusuf ${ }^{4}$, Rashida Akter \\ Khanam $^{5}$
}

\begin{abstract}
${ }^{1}$ Assistant Professor, Department of Pathology \& Microbiology, Dhaka Dental College, Dhaka, Bangladesh; ${ }^{2}$ Associate Professor, Department of Physiology, Monowara Sikder Medical College, Shariatpur, Bangladesh; ${ }^{3}$ Associate Professor, Department of Pathology \& Microbiology, Dhaka Dental College, Dhaka, Bangladesh; ${ }^{4}$ Assistant Professor, Department of Microbiology, National Institute of Neurosciences \& Hospital, Dhaka, Bangladesh; ${ }^{5}$ Assistant Professor, Department of Microbiology, Shaheed Suhrawardy Medical College, Dhaka, Bangladesh
\end{abstract}

[Received: 7 March 2019; Accepted: 20 May 2019; Published: 1 June 2019]

\begin{abstract}
Background: Newly developed KAtex test can be used as a non invasive tool for diagnosis of Kala-azar. Objectives: The aim of the present study was to compare KAtex, Bone marrow aspiration and DAT to diagnose VL. Methodology: This cross-sectional study was carried out in the Department of Microbiology at Dhaka Medical College, Dhaka, Bangladesh in collaboration with the Department of Parasitology, Institute of Epidemiology, Disease Control and Research (IEDCR), Dhaka, Bangladesh for a period of one year. Clinically suspected Kala-azar (VL) cases of different age and sex attending IEDCR, Dhaka from different Kala-azar endemic areas of Bangladesh were selected for this study. Patients having fever for more than 2 weeks, with or without splenomegaly, having history of loss of body weight following onset of fever were clinically suspected as Kala-azar cases. Microscopy and culture was performed in bone marrow (BM). KAtex was performed with urine sample. Agglutination of sensitized latex indicated presence of Leishmania donovani antigen in urine and thereby visceral leishmaniasis. No agglutination indicates absence of antigen in urine. DAT was done with serums of all cases. Result: Among 130 clinically suspected VL cases, $70(53.85 \%)$ cases were BM positive and 60(46.15\%) cases were BM negative. All the $70 \mathrm{BM}$ positive cases were positive by KAtex and DAT. Among $60 \mathrm{BM}$ negative cases, 15 were positive by KAtex and 23 were positive by DAT. The sensitivity of KAtex was $100.0 \%$ and specificity was $75.0 \%$. The sensitivity of DAT was $100.0 \%$ and specificity is $61.6 \%$. Conclusion: In conclusion, KAtex test is a good diagnostic tool for the detection of VL in comparison with DAT. [Bangladesh Journal of Infectious Diseases, June 2019;6(1):12-15]
\end{abstract}

Keywords: VL; KAtex; Bone marrow; Kala-azar; Direct agglutination test; DAT

Correspondence: Dr. Ishrat Sharmin, Assistant Professor, Department of Pathology \& Microbiology, Dhaka Dental College, Mirpur-14, Dhaka, Bangladesh: Email: dr.ishrats@yahoo.com; Cell no: +8801796248873

Conflict of interest: There is no conflict of interest to any of the authors of this article.

Funding agency: The study was not funded by any authority.

Contribution to authors: Dr. Ishrat Sharmin contributed from protocol writing upto article write up. The rest authors revised and corrected the paper.

How to cite this article: Sharmin I, Quamruzzaman AKM, Parveen R, Yusuf MA, Khanam RA. Comparison of KAtex, Bone Marrow Aspiration and DAT for the Diagnosis of Visceral Leishmaniasis. Bangladesh J Infect Dis 2019;6(1):12-15

Copyright: (02019. Sharmin et al. Published by Bangladesh Journal of Infectious Diseases. This article is published under the Creative Commons CC BY-NC License (https://creativecommons.org/licenses/by-nc/4.0/). This license permits use, distribution and reproduction in any medium, provided the original work is properly cited, and is not used for commercial purposes. 


\section{Introduction}

Visceral leishmaniasis (VL) is commonly known as Kala-azar ${ }^{1}$. It is a chronic febrile disease caused by Leishmania donovani ${ }^{2}$. The demonstration of the parasite (LD bodies) in the aspirates of the spleen, liver, bone marrow, lymph nodes is the only way to confirm VL conclusively ${ }^{3}$.

Sensitivity of bone marrow aspirate smear is estimated to be $70.0 \%$ or lower ${ }^{4}$. Sensitivity of lymph node aspirate is estimated to be $50.0 \%$ in Sudan and sensitivity of splenic aspirate exceeds $90.0 \%$. But these invasive procedures are time consuming which carries risk of hemorrhage and requires expert persons. It may be false negative if the parasite density is low $^{6}$.

Antigen detection is more specific than antibody based immunodiagnostic tests. This method is also helpful in the diagnosis of disease in cases where there is deficient antibody production ${ }^{7}$. Currently a latex agglutination test named as KAtex has been described for the detection of urinary antigens in $\mathrm{VL}^{8}$. This test is positive in active cases and it is positive within one week of infection ${ }^{9}$. KAtex becomes negative one month after completion of treatment. KAtex is simply to use, results are available within 2 minutes, it does not require any electric appliances and is thus feasible in the rural health centres. Collection of urine is acceptable to the patients. Testing of an antigen has moreover a potential for monitoring response to treatment where the antibody based tests are of no help ${ }^{9}$.

Serological tests for the detection of antibodies such as DAT have been developed in the pursuit of an alternative to demonstration of parasite. The direct agglutination test (DAT) has excellent diagnostic accuracy ${ }^{10}$. DAT is a sensitive, specific and simple test but its main disadvantage is that, it cannot readily distinguish between active disease, sub clinical infections or past infections ${ }^{8}$. Ab detection tests remain positive long after successful treatment, so can't be used to observe prognosis. Therefore, this study was undertaken to compare KAtex with BM aspiration and DAT to establish KAtex as a useful test for diagnosis of VL patients.

\section{Methodology}

This was a cross sectional study carried out in the Department of Microbiology, Dhaka Medical College, Dhaka in collaboration with the Department of parasitology in IEDCR from July 2006 to June 2007 for one year. Clinically suspected kala-azar (VL) cases of different age and sex attending IEDCR from different kala-azar endemic areas of Bangladesh were selected for this study. Patients having fever for more than 2 weeks, with or without splenomegaly, having history of loss of body weight following onset of fever were clinically suspected as kala- azar cases. Blood, Urine and bone marrow aspirations were taken with full aseptic precaution. Urine was collected in a sterile dry test tube for KAtex test. Bone marrow (BM) aspiration was done for microscopy and culture was done in N.N.N medium at $24^{\circ} \mathrm{c}$ for $1-3$ week. Data was collected in a pre designed data sheet. Then data were entered in computer and analyzed by using SPSS program. Following proper aseptic precaution blood was collected from all cases. Serum was separated by centrifugation. DAT was done with serums of all cases. Titre of $\geq 1: 3200$ was regarded as positive. Cross linking of the cells or large particles by antibody directed against surface antigens leads to agglutination ${ }^{11}$.

\section{Result}

Table 1 shows Bone marrow samples, urine samples and blood were collected from 130 clinically suspected kala-azar cases, 70 cases were bone marrow positive and 60 cases were bone marrow negative. Among 130 cases, 85(65.38\%) were KAtex positive and 93(71.54\%) cases were DAT positive.

Table 1: Comparison of Bone Marrow examination with KAtex and DAT

\begin{tabular}{|l|c|c|}
\hline BM Culture & $\begin{array}{c}\text { KAtex } \\
\text { Positive }\end{array}$ & $\begin{array}{c}\text { DAT } \\
\text { Positive }\end{array}$ \\
\hline Positive $(\mathrm{n}=70)$ & $70(100 \%)$ & $70(100 \%)$ \\
\hline Negative $(\mathrm{n}=60)$ & $15(25 \%)$ & $23(38.33 \%)$ \\
\hline Total $(\mathbf{n}=\mathbf{1 3 0})$ & $\mathbf{8 5}(\mathbf{6 5 . 3 8} \%)$ & $\mathbf{9 3 ( 7 1 . 5 4 \% )}$ \\
\hline
\end{tabular}

Table 2 shows among 130 cases, 85(65.38\%) were KAtex positive and 45(34.62\%) cases were KAtex negative.

Table: 2: Comparison of KAtex with Bone Marrow Culture

\begin{tabular}{|l|c|c|c|}
\hline \multirow{2}{*}{ KAtex } & \multicolumn{2}{|c|}{ BM Culture } & \multirow{2}{*}{ Total } \\
\cline { 2 - 3 } & Positive & Negative & \\
\hline Positive & TP 70 & FP 15 & 85 \\
\hline Negative & FN 0 & TN 45 & 45 \\
\hline Total & $\mathbf{7 0}$ & $\mathbf{6 0}$ & $\mathbf{1 3 0}$ \\
\hline
\end{tabular}

Table 3 shows that among 130 clinically suspected Kala-azar Cases, 70 Cases were bone marrow 
positive and 60 cases were bone marrow negative. Among, 130 cases, 93(71.54\%) were DAT positive and $37(28.46 \%)$ case were DAT negative.

Table 3: Comparison of DAT with Bone Marrow

\begin{tabular}{|l|c|c|c|}
\hline \multirow{2}{*}{ DAT } & \multicolumn{2}{|c|}{ BM Culture } & \multirow{2}{*}{ Total } \\
\cline { 2 - 3 } & Positive & Negative & \\
\hline Positive & 70 & 23 & 93 \\
\hline Negative & 0 & 37 & 37 \\
\hline Total & $\mathbf{7 0}$ & $\mathbf{6 0}$ & $\mathbf{1 3 0}$ \\
\hline
\end{tabular}

Table 4 shows sensitivity of KAtex is $100 \%$ and specificity of KAtex is $75 \%$. Sensitivity of DAT is $100 \%$ and specificity of DAT is $61.6 \%$.

Table 4: Sensitivity and Specificity of DAT and KAtex for diagnosis of $\mathrm{VL}$

\begin{tabular}{|l|c|c|}
\hline Variables & KAtex & DAT \\
\hline Sensitivity & $100.0 \%$ & $100.0 \%$ \\
\hline Specificity & $75 \%$ & $61.6 \%$ \\
\hline
\end{tabular}

\section{Discussion}

The study has been carried out to evaluate the performance of KAtex in the diagnosis of Kala-azar cases and to compare these results with those obtained by BM microcopy and culture and DAT. In this study, among 130 clinically suspected Kalaazar cases, 70(53.85\%) cases are BM positive and $60(46.15 \%)$ cases are BM negative. Sensitivity of $\mathrm{BM}$ aspirate smear is estimated to be $70.0 \%$ or lower ${ }^{4}$. This coincides with the result in this present study.

In the present study, among 130 cases, KAtex was positive in $85(65.38 \%$ ) cases and DAT was positive on $93(71.54 \%$ ) cases, all the $70 \mathrm{BM}$ positive cases were KAtex positive. Among $60 \mathrm{BM}$ negative cases $15(25 \%)$ were KAtex positive. In a study done by $\mathrm{Nahar}^{12}$ results of BM aspirates and KAtex were compared. In that study all the $68(100 \%) \mathrm{BM}$ positive cases were KAtex positive. Among $82 \mathrm{BM}$ negative cases $12(14.63 \%)$ were KAtex positive in that study. The result of KAtex positivity in BM negative cases in present study is higher than that of Nahar ${ }^{12}$. This is might be due to the fact that in this study, tests were done on freshly collected urine samples. In the study done by Nahar ${ }^{12}$, KAtex was done on urine samples collected from Mymensingh which were brought to Dhaka.
In another study done in Sudan, all the 15 (100\%) smear positive cases were KAtex positive. Among 47 smear negative cases $6(12.76 \%)$ were KAtex positive and $41(87.23 \%)$ were KA tex negative ${ }^{1}$. The results of KAtex positivity among smear negative cases in Sudan is lower than the present study which may be for the reason that they used both fresh and frozen urine samples.

In the present study, among 130 cases, DAT was positive in $93(71.54 \%)$ cases. All the $70(100 \%)$ BM positive cases were DAT positive. Among 60 BM negative cases 23 (38.33\%) were DAT positive. In a study done in Sudan in 1990, out of 65 smear negative patients $18(28 \%)$ were DAT positive $^{13}$. Among those 18 cases, eight were tested with the leishmanin skin test, six give a positive result suggesting past or sub clinical infection ${ }^{13}$. In another study DAT was positive in all the 20 (100\%) BM positive cases and DAT was also positive in all the $7(100 \%)$ BM negative cases ${ }^{14}$. DAT positivity among BM positive cases in the present study was similar to the study of El-Safi ${ }^{14}$. DAT positivity among BM negative cases in the present study was less than the study of El-Safi because in their study BM was done only from the DAT positive cases.

The sensitivity of KAtex is $100 \%$ and specificity of KAtex is $75 \%$ in this present study. Vilaplana showed $100 \%$ sensitivity and $96 \%$ specificity for KAtex ${ }^{15}$. In a study in Sudan showed $100 \%$ sensitivity and $87 \%$ specificity for KAtex. In separate study on 52 samples from Yemen, sensitivity of $86 \%$ and specificity of $100 \%$ for KAtex were reported ${ }^{1}$. In another study conducted by EL- Safi from Sudan showed $95.2 \%$ sensitivity and $100 \%$ specificity for KAtex ${ }^{8}$. In the present study, results of KAtex is compatible to other studies.

The sensitivity of DAT is $100 \%$ and specificity of DAT is $61.6 \%$ in this present study. In a study, done by Harith and others showed $100 \%$ sensitivity and $72.60 \%$ specificity for $\mathrm{DAT}^{16}$. In a hospital based study conducted in Sudan in 1990, DAT showed $94 \%$ sensitivity and $72 \%$ specificity ${ }^{4}$. The results of present study is similar to other studies.

\section{Conclusion}

In conclusion KAtex test for the detection of leishmania antigen in urine can be used as a non invasive tool for diagnosis of Kala-azar which has a high sensitivity in comparison with DAT. 


\section{References}

1. Kalon Biological Ltd. Alders opt, Thant's, United Kingdom, 2005

2. WHO. Technical Report Series. The leishmaniasis, report of expert Committee. No.701. Geneva : WHO: 1984:pp1-5

3. Park K. Epidemiology of communicable diseases. In: Park K editor. Park's text book of Preventive and Social Medicine. $19^{\text {th }}$ edn. M/S Banarsidas Bhanot publishers, Jabalpur, India ; 2007: pp 256-258

4. Zijlstra EE, Ali MS, Hassan AM, Towm Al, Sath M, Ghalib HB, et al. Kala-azar: a comparative study of parasitological methods and direct agglution test in diagnosis. Trans $\mathrm{R}$ Soc Trop Med hug 1992;86:505-507

5. Kager PA, Rees PH. Splenic aspiration. Review of the literature. Trop Geogr Med 1983;35:111-124

6. Goyal RK, Mohapatra TM. Superiority of DAT over ELISA as a diagnostic of sero-epidemiological tool for the diagnosis of Indian Kala-azar. Ind $\mathbf{J}$ Med Microbiol 2004;22(1):57-60

7. Rijal S, Boelaert M, Regmi S, Karki BM, Jaquel D, Singh $\mathrm{R}$, et al. Evaluation of a urinary antigen - based latex agglutination test in the diagnosis of kala-azar in eastern Nepal. Trop Med Int Health 2004;9 (6): 724

8. El-Safi SH, Haleem A, Hammad A, El- Basha, Omer A, Kareem HG, et al. Field evaluation of latex agglutination test for detection of urinary antigen in visceral leishmaniasis in Sudan. Eastern Mediterranean Health Journal 2003;9(4): 844855
9. Bahadur S, Chance M, Hommel M. Antigenuria in visceral leishmaniasis: detection and partial characterization of a carbohydrate antigen. Acta Trop 2002;82:339-348

10. Boelaert M, Ep-Safis, Mousa $\mathrm{H}$ et al. Multicenter evaluation of repeatability and reproducibility of the direct agglutination test for VL. TropMed Int Health 2004; : 31-37

11. M and PDC and IEDCR, DGHS, 1996. Laboratory diagnosis of Kala-azar (VL) with DAT- A training module ton laboratory technician, published by $\mathrm{M}$ and PDC and IEDCR, DGHS, 1996,1-19

12. Nahar K. Diagnosis of VL by detecting Leishmania antigen from urinary sample. (Thesis), BSMMU,2005

13. Zijlstra EE, Ali MS, El-Hassan AM et al. DAT for diagnosis and sero epidemiological survey of kala-azar in the Sudan. Trans R Soc Trop Med Hyg 1991;85: 474 - 476

14. El-Safi, Peters W, Evans DA. Studies on the leishmaniasis in Sudan, clinical and parasitological studies on visceral and mucosal leishmaniasis. Trans R SOC trop Med Hyg 1991; 85: 465-470

15. Vilaplana C, Blanco S, Dominiguez J, Gimenez M, Ausina $\mathrm{V}$. Non-invasive method for diagnosis of visceral leishmaniasis by a latex agglutination test for detection of antigen in urine samples. J Clin Microbiol 2004; 42 (4): 1853-1854

16. Harith AE, Kolk AH, Kager PA, et al. Evaluation of newly developed DAT for sero-diagnosis and sero-epidemiological studies of VL. Trans R SOC Trop Med Hyg 1987; 81:603-606 